\title{
Refuge
}

Canada's Journal on Refugees

revue canadienne sur les réfugiés

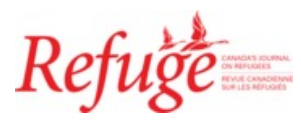

\section{La « crise migratoire " de 2015/16 en Europe : interprétation géohistorique}

\section{Étienne Piguet}

Volume 34, numéro 2, 2018

URI : https://id.erudit.org/iderudit/1055572ar

DOI : https://doi.org/10.7202/1055572ar

Aller au sommaire du numéro

\section{Éditeur(s)}

Centre for Refugee Studies, York University

ISSN

0229-5113 (imprimé)

1920-7336 (numérique)

Découvrir la revue

Citer cet article

Piguet, É. (2018). La « crise migratoire » de 2015/16 en Europe : interprétation géohistorique. Refuge, 34(2), 3-15. https://doi.org/10.7202/1055572ar
Résumé de l'article

Cet article analyse la croissance des demandes d'asile déposées sur le territoire ou à la frontière des démocraties occidentales et la " crise migratoire " qui s'en est suivie en 2015/16. Si la multiplication des foyers de violence à proximité de l'Europe a joué un rôle central, l'analyse doit être complétée par une prise en compte de trois évolutions géographiques de longue durée : le raccourcissement des distances, la crise des politiques de rétention et l'asymétrie géographique des droits. Elles permettent d'interpréter la réaction de fermeture des frontières comme une tentative de (re-)mise à distance des réfugiés dans un contexte de globalisation. Cette analyse complète par une approche géohistorique la littérature récente sur la « crise migratoire " centrée sur le rôle des partis populistes, la peur du terrorisme et les dis fonctionnements des mécanismes de solidarité.
Copyright (c) Refuge: Canada's Journal on Refugees, 2018

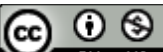

Ce document est protégé par la loi sur le droit d'auteur. L'utilisation des services d'Érudit (y compris la reproduction) est assujettie à sa politique d'utilisation que vous pouvez consulter en ligne.

https://apropos.erudit.org/fr/usagers/politique-dutilisation/ 


\title{
La «crise migratoire» de 2015/16 en Europe: interprétation géohistorique
}

\author{
ÉTIENNE PIGUET
}

\section{Résumé}

Cet article analyse la croissance des demandes d'asile déposées sur le territoire ou à la frontière des démocraties occidentales et la "crise migratoire " qui s'en est suivie en 2015/16. Si la multiplication des foyers de violence à proximité de l'Europe a joué un rôle central, l'analyse doit être complétée par une prise en compte de trois évolutions géographiques de longue durée: le raccourcissement des distances, la crise des politiques de rétention et l'asymétrie géographique des droits. Elles permettent d'interpréter la réaction de fermeture des frontières comme une tentative de (re-)mise à distance des réfugiés dans un contexte de globalisation. Cette analyse complète par une approche géohistorique la littérature récente sur la "crise migratoire" centrée sur le rôle des partis populistes, la peur du terrorisme et les disfonctionnements des mécanismes de solidarité.

\section{Abstract}

This article analyzes the increase in the number of asylum claims submitted either in-country or at the border of Western democracies and the resulting 2015-2016 "migrant crisis." Although the proliferation of outbreaks of violence near Europe has played a central role, three long-standing

(C) Etienne Piguet, 2018. This open-access work is licensed under a Creative Commons Attribution-NonCommercial 4.0 International Licence, which permits use, reproduction, and distribution in any medium for non-commercial purposes, provided the original authorship is credited and the original publication in Refuge: Canada's Journal on Refugees is cited. geographical trends must also be taken into account when considering this issue: the shrinking of geographical distance, the detention policy crisis and geographically asymmetrical rights. These trends mean that the reaction of closing borders can be interpreted as an attempt to keep refugees at a distance once again, against a background of globalization. The analysis finishes with a geohistorical approach to recent literature on the "migrant crisis," focusing on the role of populist parties, the fear of terrorism, and the dysfunctionality of solidarity mechanisms.

$\mathrm{P}$ lus de 2,5 millions de demandes d'asile ont été déposées en Europe entre 2015 et 2016 selon EUrostat ${ }^{1}$. Des milliers dêtres humains ont perdu la vie sur le chemin de l'exil². Même si l'immense majorité des réfugiés restent dans les pays du Sud 3 , cette «crise migratoire» a suscité des tensions considérables en Europe. Les diagnostics politico-médiatiques sont fortement polarisés. Pour les uns, c'est l'attrait de l'Europe pour des migrants économiques et son laxisme dans le contrôle des frontières qui sont en cause. Pour les autres, c'est l'égoïsme, le barricadage des voies d'entrées légales et le mépris des droits humains qui marquent la faillite morale de l'Europe. Parmi les chercheurs, trois approches de la crise peuvent être identifiées. La première,

Cette œuvre en libre accès fait l'objet d'une licence Creative Commons Attribution-NonCommercial 4.o International License, laquelle autorise l'utilisation, la reproduction et la distribution de l'œuvre sur tout support à des fins non commerciales, pourvu que l'auteur ou les auteurs originaux soient mentionnés et que la publication originale dans Refuge: revue canadienne sur les réfugiés soit citée. 
sur laquelle nous reviendrons, porte sur les causes de fuite et les itinéraires des migrants ${ }^{4}$, la deuxième propose une réflexion critique sur la manière dont le phénomène a été appréhendé, les catégories (réfugiés, migrants, demandeurs d’asile, etc...) utilisées pour le décrire et les violations des droits humains ${ }^{5}$. Enfin, la troisième approche adopte une perspective politologique sur les raisons du manque de cohérence de l'Europe et la crise politique qui s'en est sui$v^{6}{ }^{6}$ (difficultés à intégrer les flux migratoires précédents, inégalités sociales croissantes, craintes face à la globalisation, islamophobie, peur du terrorisme, montée des partis xénophobes, etc.).

Dans cet article, nous tentons de de compléter ces analyses en nous concentrant sur les transformations structurelles sous-jacentes à la croissance des demandes d'asile. Nous laissons donc de côté les arrivées de migrants sans papiers et les autres formes d'immigration, même si elles sont certainement aussi concernées par les évolutions structurelles décrites. Notre positionnement épistémologique est celui de la géohistoire ${ }^{7}$ puisque nous chercherons, sur une moyenne durée, à comprendre en quoi les espaces de circulation géographique des migrations se sont transformés, ouvrant ou fermant des opportunités de protection à longue ou courte distance pour les victimes de violence. D'un point de vue méthodologique, notre approche ne cherche pas à valider ou rejeter telle ou telle hypothèse. Nous cherchons, pour paraphraser Alejandro Portes, à proposer une interprétation, étayée et convaincante des enchainements de causes multiples du phénomène étudié ${ }^{8}$.

Notre thèse centrale est que, malgré certains points communs avec des crises antérieures (ampleur des déplacements, manque de coordination entre les pays d'accueil, peurs et xénophobie) $)^{9}$, la situation actuelle marque une rupture dans l'articulation des territoires, de la souveraineté et des droits dans un contexte de globalisation. La crise a culminé en 2015/2016 en raison de la guerre civile syrienne, mais elle trouve son origine dans des évolutions plus anciennes. Laccalmie fragile qui prévaut depuis 2017 s'explique par la détermination de l'ue à réinstaller le régime de mise à distance des réfugiés en place depuis l'après-guerre, mais les fondamentaux d'un profond déséquilibre restent en place.

\section{Historique d'une crise inédite}

Commençons par un ordre de grandeur historique pour les dix principaux pays d'accueil en Europe (Graphique 1). Lampleur record des demandes d'asile de 2015/16 y apparaît clairement ${ }^{10}$. D'autres épisodes de forts afflux doivent cependant être relevés. Les années 1991, 92 et 93 voient arriver en Europe - et principalement en Allemagne - de très nombreux ressortissants d'ex-Yougoslavie fuyant les crises en Slovénie puis en Croatie et surtout en Bosnie. Beaucoup devront rentrer dès 1995, mais on estime que 350 ooo seront accueillis à long terme ${ }^{11}$. Entre 1988 et 1993, la difficile transition post-Ceaucescu ${ }^{12}$ pousse 350000 Roumains à déposer une demande d'asile en Europe, le plus souvent sans succès ${ }^{13}$. Dix ans plus tard, les demandes d'asile avoisinent 400000 quatre années consécutives, principalement en raison de la guerre au Kosovo ${ }^{14}$. Une part importante des Kosovars seront acceptés, mais au seul bénéfice de permis temporaires et ils seront nombreux à devoir regagner leur pays au cours des années $2000^{15}$.

Il ressort de ces chiffres que, sans être totalement horsnorme, les années 2015/16 sont bien exceptionnelles. Ce constat reste valide si l'on remonte avant 1980 aux épisodes d'exode hongrois (200 ooo départs de réfugiés en $1956 / 7^{16}$ ) et tchécoslovaque (170 ooo départs en $1968 / 9^{17}$ ). Le cas des boat-people indochinois, dès 1975, est comparable à 2015/16 en termes de départs et de drames humains (3 millions de départs, des milliers de morts par naufrage), mais seule une petite minorité de réfugiés $(185000)$ sera réinstallée en Europe $^{18}$.

Proportionnellement à la population de l'Europe (500 millions d'habitants), larrivée de 2.5 millions de demandeurs d'asile en 2015/16 équivaut à un taux annuel de $0.25 \%$. On est donc loin des «flots» ou des «déferlements» décrits par certains médias. Plusieurs pays dont l'Allemagne (1\%) et la Suède $(1.5 \%)$ concentrent cependant les arrivées et, pour la démographie historique, un taux d'immigration annuel de $1 \%$ constitue un chiffre fort élevé ${ }^{19}$. Il correspond aux plus fortes arrivées migratoires en Europe des années 1960 et à celles de la fin du xixe siècle aux Etats-Unis ${ }^{20}$. Il est donc compréhensible que, par-delà des réactions xénophobes condamnables, certains gouvernements se soient inquiétés de lampleur du phénomène.

Pour certains observateurs, la crise migratoire s'expliquerait par une proportion élevée de migrants économiques sans besoins de protection qui viendraient saturer les systèmes d'asile. Il est vrai que cette explication a parfois pu s'appliquer. Ainsi au début des années nonante les demandes d'asile de dizaine de milliers de Roumains en Allemagne avaient peu de fondements autres quéconomiques. Plus récemment, de nombreux migrants des Balkans ont tenté leur chance sans que leurs pays ne connaissent des situations de violence ou de violation des droits humains marquées. Au cours des années récentes cependant le tableau ci-dessous montre que les origines de la majorité des demandeurs d'asile correspondent à des pays où les violences et les violations à large échelle des droits humains sont largement documentées.

Dans d'autres cas (Afrique sub-saharienne, Pakistan, Balkans), il est difficile de tracer une séparation claire entre catégories de migrants ${ }^{21}$. De manière générale cependant, les études quantitatives systématiques sur les déplacements des 


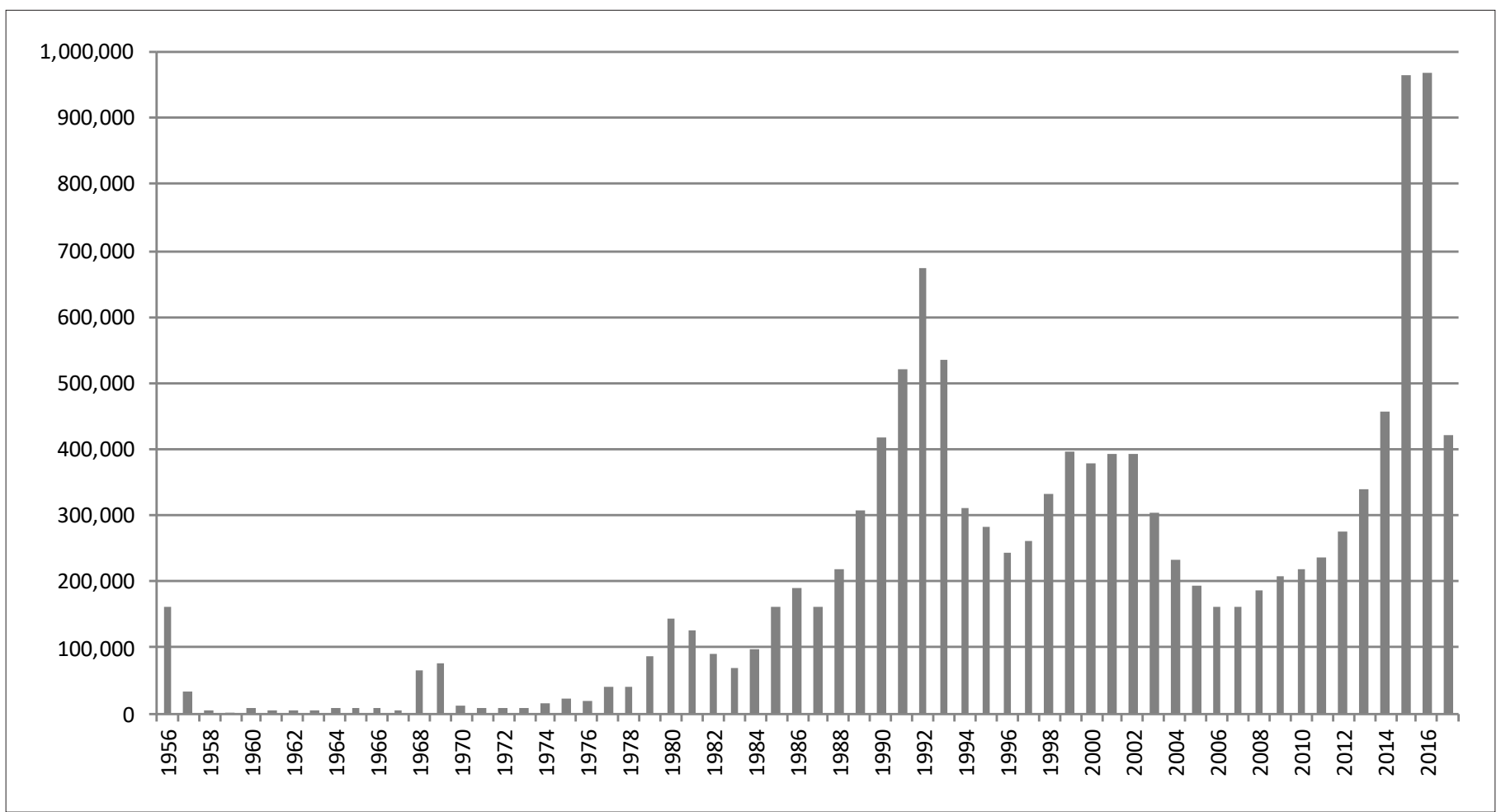

Graphique 1. Demandes d'asile dans 10 pays d'Europe 1956-2017

Source: UNHCR/Eurostat (Demandes déposées pour la 1e fois) - Avant 1980 propres estimations basées sur UNHCR

Tableau 1. Principales origines des demandes d'asile dans I'UE+

\begin{tabular}{|c|c|c|c|c|c|}
\hline \multicolumn{2}{|c|}{2011} & \multicolumn{2}{|c|}{2012} & \multicolumn{2}{|c|}{2013} \\
\hline Afghanistan & 24235 & Afghanistan & 23385 & Syrie & 49155 \\
\hline Pakistan & 14985 & Syrie & 22260 & Russie & 35810 \\
\hline Iraq & 13485 & Pakistan & 17405 & Erythrée & 19930 \\
\hline Somalie & 13260 & Somalie & 15725 & Pakistan & 19450 \\
\hline Syrie & 124750 & Syrie & 377910 & Syrie & 337505 \\
\hline Erythrée & 45885 & Afghanistan & 193015 & Afghanistan & 186530 \\
\hline Afghanistan & 39135 & Iraq & 126810 & Iraq & 128530 \\
\hline Kosovo & 34590 & Kosovo & 67535 & Pakistan & 47780 \\
\hline
\end{tabular}

Source: Eurostat (Demandes déposées pour la 1e fois) - Union Européenne + Suisse et Norvège 
demandeurs d'asile confirment que la violation des droits humains constitue le facteur de fuite le plus important ${ }^{22}$.

Historiquement, guerres et violences sont donc les principales causes de fluctuation des demandes de protection ${ }^{23}$ en Europe, mais d’autres évolutions, sans rapport direct avec les motifs de fuite, expliquent leur croissance tendancielle et culminent en 2015. Force est en effet de constater que d'innombrables épisodes de violence ont marqué l'après second guerre mondiale sans générer, à distance égale, des déplacements de population significatifs vers l'Europe. En 1967, la guerre du Biafra déplace 2 millions de personnes au Nigeria, en 1979, l'invasion de l'Ethiopie par la Somalie déplace 600 ooo réfugiés, en 1985 la guerre d'indépendance en Erythrée fait 1.2 millions de réfugiés ${ }^{24}$, en 1992 la guerre civile au Mozambique fait 6 millions de déplacés, en 1994 le génocide au Rwanda 3.5 millions. En 2007 le HCR estimait à 2.2 millions le nombre d'Irakiens ayant fui le pays principalement vers ses voisins.

Il est donc nouveau que se dirigent vers l'Europe, de manière spontanée, désorganisée, et avec un coût humain intolérable des centaines de milliers de personnes en quête de protection. Trois explications conjointes peuvent être envisagées: le raccourcissement des distances, la crise des politiques de rétention et l'asymétrie géographique des droits.

\section{Rapprochement des crises et connections croissantes}

La distance physique a constitué historiquement un frein majeur pour les personnes en quête de protection ${ }^{25}$. Ces dernières se déplacent encore aujourd'hui le plus souvent sur de courtes distances et privilégient les pays limitrophes ou, s'ils noffrent pas une protection suffisante, les premiers pays sûrs ${ }^{26}$. On peut relever à ce sujet que les troubles politiques ayant donné naissance à la crise migratoire de 2015 se trouvent plus proches du territoire européen que ce nétait le cas lors de nombreuses autres crises du passé, en particulier l'exode des boat-people indochinois. La Syrie, en particulier, se trouve aux marges immédiates de l'Europe. C'est là une première explication à l'ampleur des arrivées. De manière beaucoup plus générale cependant, ce sont les distances elles-mêmes que la globalisation a, en quelque sorte, «raccourcies ${ }^{27}$. Les populations à protéger se sont par conséquent «rapprochées»; ce qui dès les années 1980 a pris par surprise les pays signataires du protocole de 1967 élargissant au monde entier la Convention de 1951 auparavant limitée à l'Europe ${ }^{28}$. Ainsi, selon Gil Loescher, "The 1980s rudely shook the industrialized countries out of their old notions of insularity from the world's refugee problems. Apart from the occasional ballet dancer, rocket scientist, or merchant marine seaman from the Soviet bloc, political asylum had been an exceptional event for the West. (...) Western governments never envisaged large-scale population movements from the Third World. The developed world was simply too distant ${ }^{29}$. Depuis lors, le phénomène de rapprochement s'est accéléré. Quelles en sont les composantes?

\section{Connexions croissantes}

La première est l'accroissement des flux d'information qui contraste avec l'isolement des zones de crise de la guerre froide. Il semble raisonnable de postuler que les groupes et les individus soumis à des persécutions ou à des violences sont aujourd'hui beaucoup mieux informés sur les perspectives d'accueil et les modalités de déplacement. Il s'agit là d'un processus de longue durée - En 1938 un auteur fait déjà une observation similaire ${ }^{30}$ - mais l'accélération récente reste spectaculaire. Une étude chiffre ainsi à $86 \%$ la proportion des jeunes réfugiés syriens possédant un téléphone mobile dans les camps de premier asile ${ }^{31}$ et la Banque mondiale observe en Afghanistan ${ }^{32}$ un taux d'abonnement au téléphone mobile de 62\% en 2015, en Irak de 92\% (Figure 2). Les moyens de télécommunication facilitent l'organisation du déplacement ${ }^{33}$ et parfois les secours ${ }^{34}$. En 2015 on a ainsi vu apparaitre des applications pour smartphones spécifiquement destinées à la traversée des pays des Balkans ${ }^{35}$ ou à l'arrivée en Allemagne ${ }^{36}$. Plus récemment sont apparues des applications permettant de contacter un traducteur volontaire via Facebook ${ }^{37}$. Limplication croissante de la société civile dans le support aux réfugiés contribue aussi à ce rapprochement par des actions d'assistance et de sauvetage dans les zones de transit.

\section{Moyens financiers et diasporas}

Une deuxième évolution qui tend à faciliter l'accès à l'Europe est l'accroissement des moyens financiers des ménages de nombreux pays dorigine. Si les inégalités se creusent, des centaines de millions de personnes sont néanmoins parvenues durant la dernière décennie à sortir de l'extrême pauvreté. Selon certaines estimations, six cent soixanteneuf millions de personnes auraient passé de la catégorie «pauvre» à «bas revenu ». Cette évolution touche en premier lieu des pays tels que la Chine, mais l'Afrique a aussi connu une importante diminution de la pauvreté, y compris dans certains pays touchés par la violence: en Ethiopie la part des pauvres aurait diminué de 26 points de pourcentage, au Nigeria de $18^{38}$. Mobiliser quelques milliers de dollars afin de financer un voyage était totalement hors de portée d'une écrasante majorité des populations du Sud il y a quelques décennies. C'est désormais possible pour un plus grand nombre, pour qui s'accroît ce qu'Arjun Appadurai appelle la "capacité d'espérer ${ }^{39}$. L'exode syrien marqué par une forte représentation de la classe moyenne est un bon exemple de ce phénomène $\mathrm{e}^{40}$, à comparer avec la guerre du Biafra à la fin 


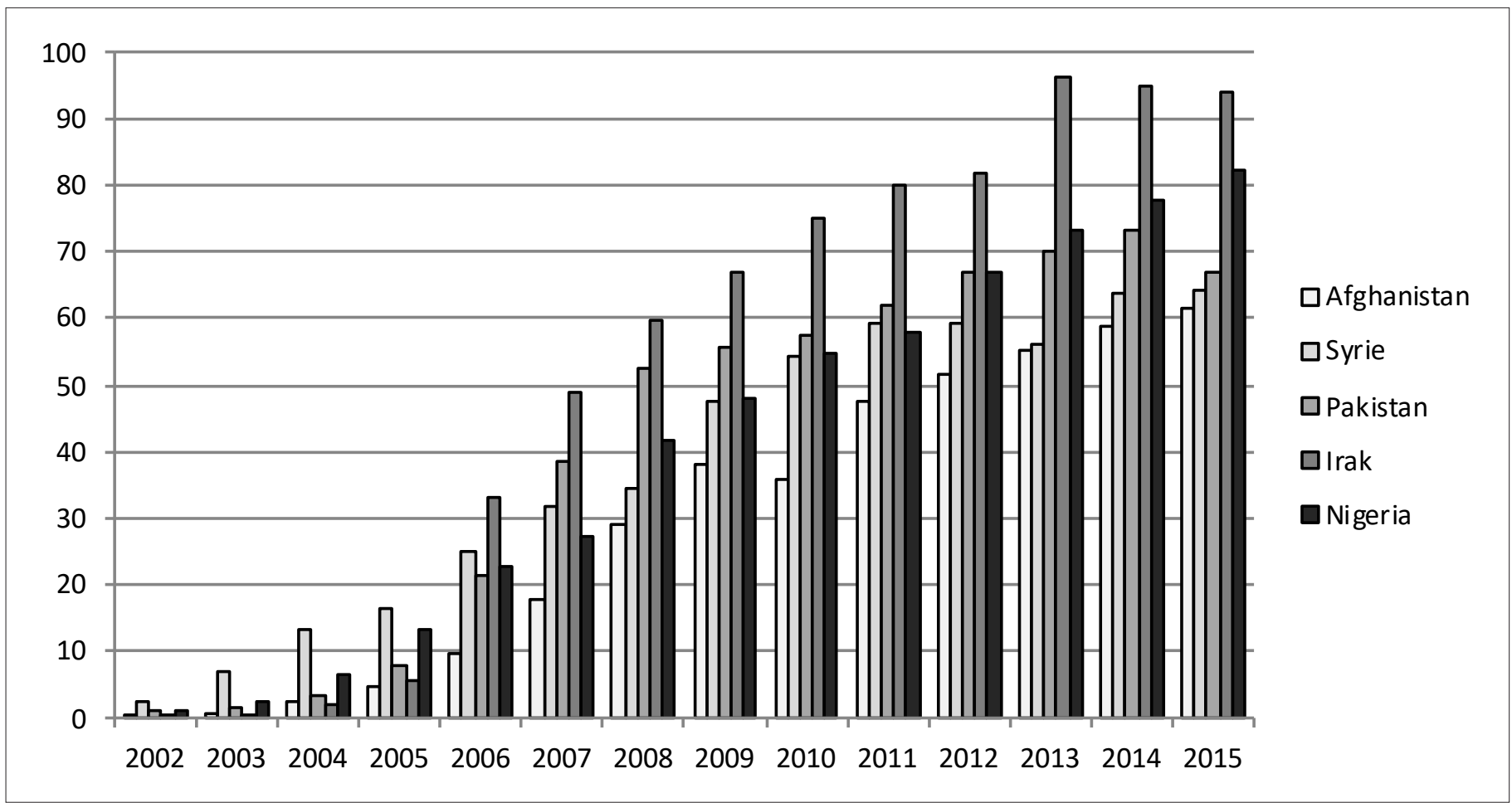

Graphique 2. Abonnements de téléphonie mobile pour 100 habitants

Source: Banque mondiale

des années soixante ou, aujourd'hui encore, avec l'exode des Rohingyas du Myanmar confinés au Bangladesh.

Lexistence de diasporas accentue encore les liens entre zones dorigine et destinations potentielles et facilite le financement des déplacements. Dans certains cas des canaux migratoires préexistants, issus de la migration de travail, se réactivent en cas de crise, comme ce fut le cas entre la Suisse et le Kosovo dans les années 2000, et permettent la fuite d'importantes populations. La fermeture de certaines voies d'accès économiques ou familiales peut aussi renforcer l'incitation à emprunter celle de l'asile.

\section{Fin du contrôle à la sortie et réseaux de passeurs}

Une troisième évolution géographique tient au déclin du «contrôle à la sortie» qui était l'une des grandes caractéristiques du régime migratoire de la guerre froide ${ }^{41}$. Ainsi de nombreux pays empêchaient leurs citoyens de quitter le territoire et considéraient le départ comme un délit. Bien que dénoncée par les pays démocratiques comme une violation des libertés, cette interdiction évitait tout exode massif qui aurait mis en péril le régime d'asile international. A l'heure actuelle, la fuite est parfois difficile, voire sévèrement réprimée, mais la fragmentation politique qui caractérise de nombreuses zones de conflit permet tout de même des exodes transfrontaliers substantiels. Dans certaines régions, le pouvoir central n'a plus ni la volonté, ni les ressources, pour exercer un contrôle effectif sur ses ressortissants. En Erythrée, s'il n'hésite pas à faire tirer sur les fugitifs, le gouvernement s'accommode ensuite fort bien du départ des survivants en imposant officiellement aux expatriés une taxe sur les revenus acquis à l'étranger ${ }^{42}$. Le départ de réfugiés qui durant la guerre froide était vu par les pays d'accueil et dorigine comme un enjeu symbolique majeur pour démontrer la supériorité d'un système politique sur l'autre $a$, dans une large mesure, perdu ce statut ${ }^{43}$ ce qui diminue l'incitation au contrôle à la sortie, tout comme dailleurs à l'accueil généreux ${ }^{44}$.

Enfin, la mise en place de réseaux de plus en plus professionnels de passeurs est une quatrième évolution qui contribue à faciliter - tout en rendant parfois plus dangereux - les déplacements. Si elle est souvent mise en avant dans le discours politique, l'activité des passeurs reste cependant plus une conséquence des autres évolutions et des besoins de protection qu'une cause en $\mathrm{soi}^{45}$.

De manière interdépendante, les évolutions que nous venons dévoquer contribuent à accroître l'effectif des personnes pouvant envisager de tenter leur chance vers l'Europe. Sans abolir les freins au déplacement, elles définissent un 
cercle géographique élargi d'accès possible qui inclut désormais la Syrie, l'Afghanistan, le Pakistan, la corne de l'Afrique et l'Afrique sub-saharienne. Ces zones de crises prépondérantes dans l'exode vers l'Europe correspondent à un périmètre d'environ 3000 kilomètres tandis qu'au-delà, l'Afrique des Grands lacs, l'Amérique du Sud ou le Myanmar restent trop distants pour générer des migrations substantielles vers l'Europe. D'autres régions d'accueil, l'Australie, les Etats-Unis et le Canada connaissent des évolutions comparables mais des conditions géographiques et des réponses politiques contrastées. Les gouvernements européens tentent de lutter contre lélargissement des provenances en rendant plus difficile le franchissement des distances par des mesures de contrôle, des sanctions aux transporteurs de personnes sans documents de voyage, etc. Ces tentatives de rétention qui ne sont pas nouvelles sont cependant elles aussi en crise.

\section{Affaiblissement du pacte de rétention}

Un deuxième élément d'explication de la crise actuelle tient à l'affaiblissement, de ce que l'on pourrait appeler le "pacte de rétention" - en anglais de containment - informellement mis en place après la seconde guerre mondiale et durant la décolonisation lorsque les pays du Nord commencèrent à se préoccuper du sort des populations déplacées à léchelle mondiale.

\section{La répartition des tâches mise en cause}

Pour Luc Legoux: "A la fin des années soixante, avec l'extension de la guerre froide à l'ensemble du monde, une répartition des tâches est instaurée (...). L'Occident accueille toujours un petit flux de réfugiés politiques essentiellement en provenance du monde communiste, les pays du Sud hébergent les flux massifs de réfugiés des guerres locales liées au conflit entre les deux blocs. ${ }^{46}$. C'est le HCR qui assure dans une large mesure l'assistance des réfugiés dans des camps dans un contexte délargissement de son mandat voulu par le Haut-commissaire Felix Schnyder ${ }^{47}$ et grâce au financement des pays du Nord ${ }^{48}$. Dans les cas trop problématiques, la réinstallation est une voie par laquelle une minorité de réfugiés se trouve finalement transférée au Nord. Plusieurs auteurs ${ }^{49}$ ont mis en évidence lexistence de cette répartition des tâches basée sur l'idée d'une différence de nature entre réfugiés des pays de l'Est, considérés à priori comme "politiques", et réfugiés du Sud, considérés collectivement comme fuyant la violence. De nombreux auteurs ont critiqué le caractère artificiel de cette distinction que Bhupinder Chimni qualifie de «myth of difference ${ }^{50}$.

La répartition des tâches entre pays d'accueil au Sud et bailleurs de fonds au Nord est évidemment toujours en vigueur, comme en témoignent les effectifs très supérieurs de réfugiés hébergés dans les pays du Sud et l'ampleur des activités d'assistance du $\mathrm{HCR}^{51}$. Le contexte favorable des années 60-70 - passé commun de lutte pour la décolonisation au Sud, ampleur de l'aide financière du Nord et relative prospérité de certains états décolonisés ${ }^{52}$ - s'est cependant transformé avec la fin de la guerre-froide et les pays occidentaux rechignent souvent à financer les activités d'assistance ${ }^{53}$. La Guinée, le Malawi et le Pakistan qui hébergeaient de nombreux réfugiés s'en plaignaient déjà dès les années $80-90^{54}$.

La crise syrienne est emblématique du désengagement des pays du Nord pour assurer la contrepartie financière de l'hébergement au Sud. Ainsi on a constaté quelles difficultés le Programme Alimentaire Mondial (PAM), le HCR et les autres organismes d'aide ont eues à financer leurs activités durant la crise. En 2014 le PAM annonçait avoir dû couper de $30 \%$ l'assistance aux réfugiés syriens du Liban ${ }^{55}$. De même, quelques semaines avant l'explosion des arrivées de réfugiés dans les îles grecques en 2015, un rapport conjoint de 200 organisations d'assistance annonçait que dans les zones de premier accueil, 1,6 million de réfugiés syriens avaient vu leur aide alimentaire réduite, que 750000 enfants nétaient pas scolarisés et que les services de santé vitaux étaient trop chers pour beaucoup, y compris 70000 femmes enceintes ${ }^{56}$. De manière générale, si les contributions au HCR ont cru en valeurs absolues au cours des années récentes ${ }^{57}$, cette croissance n'a clairement pas suivi celle des besoins de protection.

Bien sûr, mobiliser au Nord des fonds d'assistance pour les réfugiés dans les pays du Sud a toujours été difficile - le HCR fut confronté à de tels défis en Indochine ${ }^{58}$ - mais le contexte de guerre froide et de compétition entre les blocs y était beaucoup plus favorable qu'aujourd'hui. Selon Jeff Crisp, la démocratisation observée dans de nombreux pays du Sud a par ailleurs rendu plus difficile l'acceptation de politiques d'accueil et d'hébergement de grande ampleur ${ }^{59}$, transposant du Nord au Sud la tension bien connue entre processus démocratiques et accueil libéral des réfugiés ${ }^{60}$. On peut ajouter que le traumatisme du massacre de Srebrenica en 1995 a sans doute aussi grandement freiné les velléités de créer des zones protégées à l'intérieur même des régions en conflits comme cela avait été fait lors de l' "Operation Provide Comfort» de 1991 pour les Kurdes d'Irak afin d'éviter leur entrée en Turquie et leur éventuelle migration vers l'Europe ${ }^{61}$.

\section{Fermeture des voies d'accès protégées}

Nous l'avons relevé ci-dessus, la répartition des tâches instaurée entre le Sud et le Nord dans l'accueil des réfugiés comprenait aussi certaines modalités permettant d'accéder au territoire des pays du Nord ou de faire valoir des motifs de protection à distance. Une première passait par les ambassades : l'asile diplomatique théorisé par Grotius au Xvıre siècle, a longtemps donné aux bâtiments diplomatiques un statut extraterritorial ${ }^{62}$ permettant de demander protection sans 
quitter son propre pays. En Suisse, la possibilité de déposer une demande d'asile en ambassade, tacitement reconnue dans l'après-guerre, a été formellement inscrite dans la première loi sur lasile de 1979. Elle trouvait son équivalent dans de nombreuses législations européennes de l'époque.

En second lieu, les programmes de réinstallation menés principalement sous légide du Haut Commissariat des Nations unies pour les réfugiés (HCR) consistaient, pour un pays d'accueil, à accepter un contingent de personnes sélectionnées par le HCR dans des zones de premier exil. Les exemples historiques sont ici nombreux,du plan d'action international pour l'Indochine entre 1985 et $1997^{63}$ au pont aérien mis en place pour le Kosovo en 1999. Dans ce dernier cas, près de 100 ooo réfugiés furent évacués des Balkans vers l'Europe par les forces de l'otan puis répartis vers 28 pays d'accueil ${ }^{64}$.

Les deux instruments que nous venons d'évoquer ont permis dans le passé à des centaines de milliers de personnes de bénéficier d'une protection. Si les programmes de réinstallation sont toujours en vigueur, leur rythme de croissance est loin d'avoir suivi celui les besoins de protection. En 2016 les réinstallations ne représentaient que $3 \%$ des décisions de protection en Europe: tous les autres réfugiés étaient arrivés par leurs propres moyens ${ }^{65}$.

Laccès aux procédures d'asile dans les ambassades s'est lui aussi considérablement restreint. En 2002, on recensait sept pays européens (l'Autriche, le Danemark, la France, les Pays-Bas, l'Espagne, la Grande-Bretagne et la Suisse) offrant cette possibilité $^{66}$. Depuis lors, tous l'ont abolie ou fortement restreinte ${ }^{67}$.

Ce chapitre nous permet de conclure que les pays du Nord ont de longue date cherché à tenir à distance les réfugiés potentiels des pays du Sud. La forteresse Europe n'est, à cet égard, pas nouvelle, mais elle a longtemps reposé sur un pacte tacite qui impliquait, en contrepartie à la fermeture, d'importantes contributions financières et le maintien de voies d'accès alternatives.

La fragilisation du pacte de rétention et le rapprochement des crises contribuent désormais à accroître les besoins de protection exprimés sur le territoire européen. Reste cependant à comprendre ce qui peut pousser un nombre croissant de migrants à prendre le risque d'un déplacement souvent meurtrier. Lasymétrie croissante entre les droits pouvant être associés à la présence sur le territoire européen et ceux dont peut se prévaloir une personne de l'extérieur l'explique.

\section{Asymétrie géographique des droits}

Largument développé ici nuance la thèse souvent avancée d'une dégradation générale des conditions d'accueil des demandeurs d'asile en Europe. Si l'attitude de certains gouvernements et la récupération par des mouvements xénophobes, ont contribué à crisper une partie de la population vis-à-vis des demandeurs d'asile et si de multiples politiques de dissuasion ont été mises en place, une évolution concomitante allant vers des droits plus étendus pour les réfugiés se manifeste depuis une vingtaine d'années ${ }^{68}$. Comme le relèvent Thielemann \& Hobolth «In many developed countries, material reception conditions have been improved, the definition of what constitutes protection needs has been widened, procedural safeguards in the refugee determination process and against the removal of those not qualifying for refugee protection have been strengthened ${ }^{69}$. Deux évolutions sont particulièrement remarquables, lélargissement de la définition et la judiciarisation des procédures.

\section{Elargissement de la définition du réfugié}

La définition du réfugié figurant dans la Convention de 1951 implique une persécution individuelle. Si elle a d'emblée été utilisée de manière flexible en fonction des enjeux politiques du moment ${ }^{70}$, cette définition a progressivement été considérablement élargie dans les législations européennes. Pour Matthew Gibney: "As restrictive measures have developed across Europe and North America, the legal grounds on which individuals can claim asylum have expanded significantly. Far from confining themselves simply to the 1951 Convention on Refugees, western countries now accept a range of human rights grounds as a basis for asylum ${ }^{71}$. De nombreux pays ont ainsi considéré les persécutions non-étatiques et les violences généralisées comme pouvant justifier loctroi d'une protection. Le HCR a joué un rôle important dans cet élargissement à travers les clarifications successives données aux principes directeurs sur la protection entre 2002 et 2016. La dernière clarification de décembre 2016 considère ainsi que la Convention est «directement applicable aux civils déplacés par des situations de conflits armés et de violence ${ }^{72}$ et reflète la position du HCR durant la crise syrienne. Auparavant, le HCR avait été plus nuancés, par exemple pour les Tamouls du Sri-Lanka au milieu des années quatre-vingt ${ }^{73}$, et pour les ressortissants d'ex-Yougoslavie au début des années nonante ${ }^{74}$. Dans les deux cas, le HCR se fit - entre autre pour des raisons pragmatiques d'acceptabilité par les états d'accueil - l'avocat d'un accueil temporaire sans reconnaissance formelle du statut de réfugié.

L'une des conséquences de lélargissement de la définition des personnes pouvant bénéficier du statut de réfugié semble avoir été, dès 2013, le déclin de la proportion des formes de protection subsidiaires et souvent temporaires. Ces statuts de deuxième ordre avaient été mis en place dans un but de dissuasion dans les années nonante à l'instigation de l'Allemagne et sétaient rapidement appliqués à 50\% des décisions de protection au sein de l'UE ${ }^{75}$. 


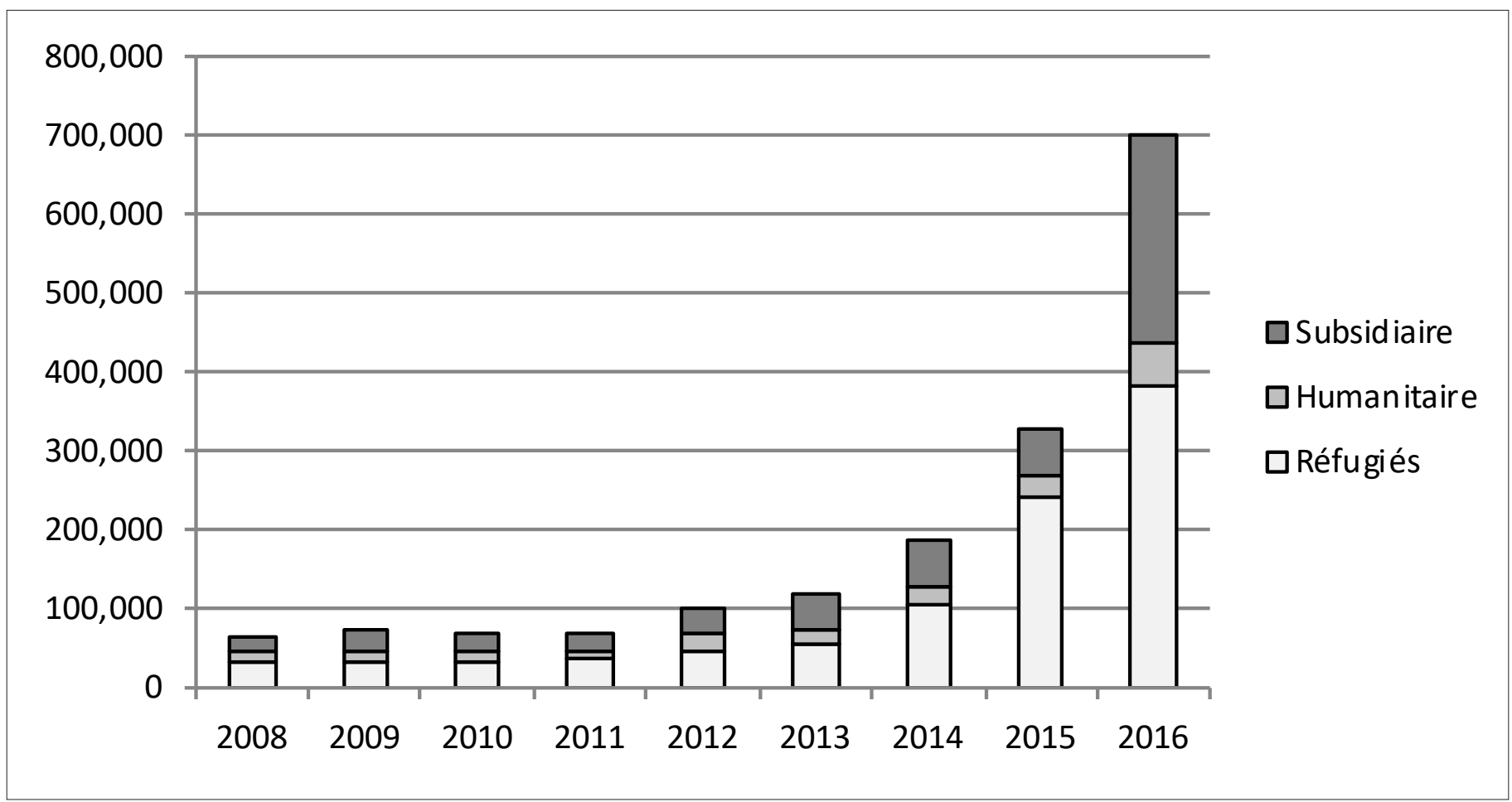

Graphique 3. Décisions positive de $1^{\mathrm{e}}$ instance dans l'UE+ selon statut (val. absolues)

Source: Eurostat

Leur proportion tombe à $20 \%$ environ en 2015 avant d'augmenter à nouveau en 2016, probablement suite à la volte-face restrictive de plusieurs pays d'Europe ${ }^{76}$.

\section{Judiciarisation}

La deuxième évolution importante est le transfert partiel des compétences de décision de la sphère administrative à la sphère judiciaire. Les tribunaux jouent ainsi un rôle croissant dans la détermination des statuts de protection et tendent à contrecarrer les tendances restrictives des administrations étatiques ${ }^{77}$. A l'octroi discrétionnaire de l'asile par l'Etat, longtemps dominant, se substituent des procédures basées sur des droits clairement spécifiés et à caractère universel. Cette évolution s'inscrit dans une dépolitisation de lasile sur la longue durée: les critères d'accueil ne sont plus liés comme au temps des proscrits du xixe siècle ou de la guerre froide à un jugement porté par un Etat d'accueil sur un Etat d'origine. Désormais, ces considérations sont supplantées par la situation humanitaire individuelle des personnes accueillies. Matthew Price parle de dépolitisation ${ }^{78}$ et d'humanitarianisation de l'asile ${ }^{79}$. Une jurisprudence importante s'est aussi développée au niveau de la Cour européenne des droits de l'homme (CEDH) et étend certaines protections, jusqu'ici réservées aux réfugiés, aux personnes ne pouvant se prévaloir de ce statut ${ }^{80}$. Comme le relève Alexander Betts: "The most high-profile cases have shown that those who are not includable or are excludable under international refugee law may nevertheless be entitled to international protection if they face, for example, the prospect of torture or cruel, inhuman, and degrading treatment on their return ${ }^{81}$. Ainsi, le Jugement Salah Sheek (2007) de la CEDH considère pour la première fois qu'un renvoi peut être interdit en raison d'une situation de violence généralisée sans que la situation individuelle de la personne concernée ne diffère de celle du reste de la population ${ }^{82}$.

\section{Asymétrie}

Lélargissement de la définition du réfugié et la judiciarisation des procédures ont pour conséquence un élargissement des perspectives de protection et de séjour qui souvrent aux candidats à l'asile en Europe. Dans le même temps, la présence sur le territoire du pays d'accueil est requise pour en bénéficier tandis que les voies d’accès se raréfient et que les restrictions se multiplient dans le cadre de ce que l'on peut appeler un régime de non-entrée ${ }^{83}$. Issu des traités de Westphalie (1648) et centré sur l'Etat-nation, le droit d'asile reste en effet marqué par cette condition cardinale: Il faut, pour être protégé, être à l'intérieur du territoire ${ }^{84}$. Lasymétrie entre 


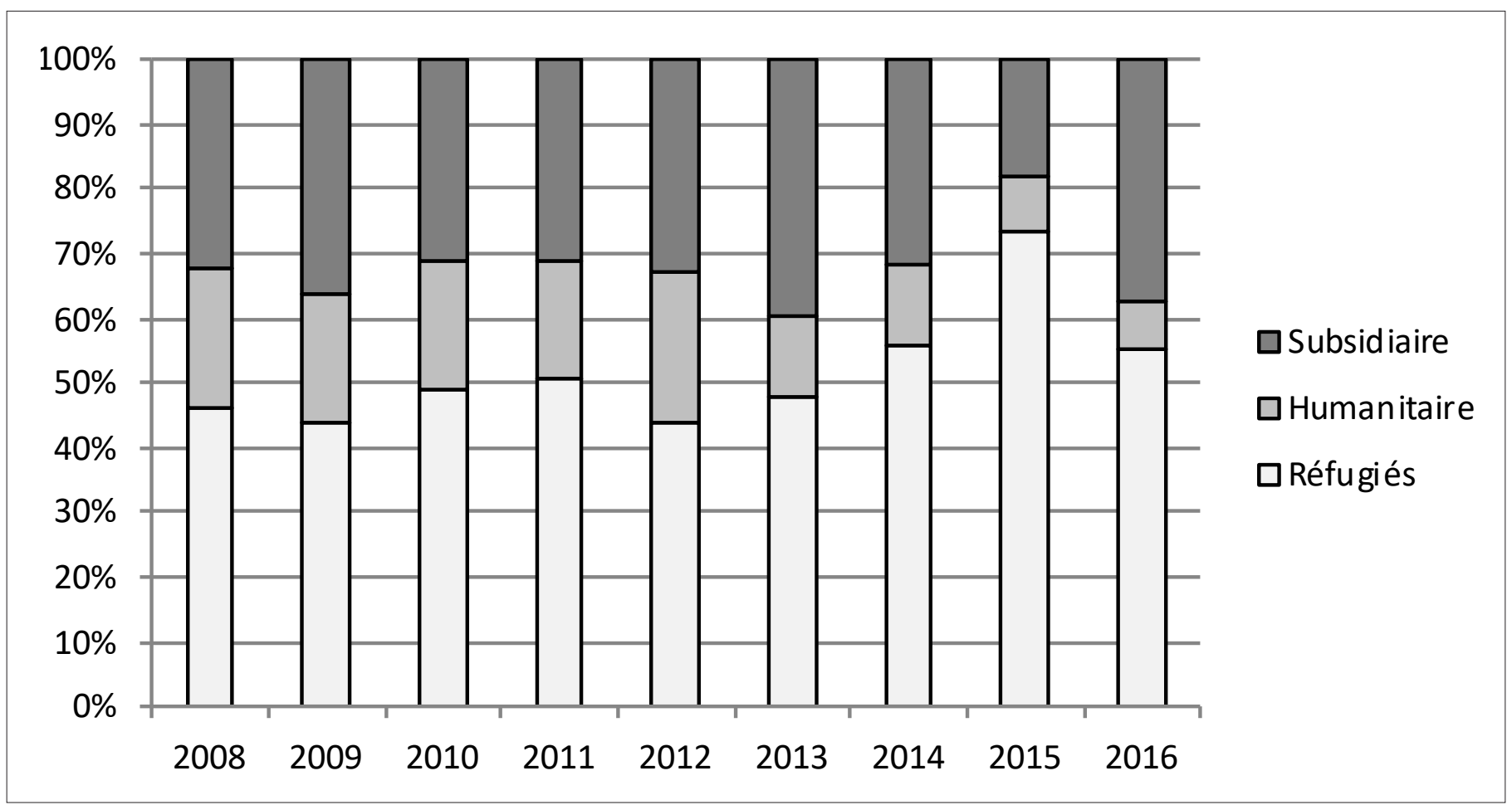

Figure 4. Décisions positive de $1^{\mathrm{e}}$ instance dans l'UE+ selon statut (pourcentages)

Source: Eurostat

les pays où s'appliquent le droit à la protection et le reste du monde s'en trouve accrue. Elle a été confirmée récemment par un jugement de la Cour de justice de l'ue déboutant une famille syrienne qui avait tenté de demander un visa pour fuir la ville assiégée d'Alep alors quelle réunissait clairement les qualités qui lui auraient permis d’obtenir un statut de séjour si elle avait pu le demander en Europe ${ }^{85}$. La clause de non-refoulement, désormais généralisée par la $\mathrm{CEDH}^{86}$, tend à accroître cette asymétrie des droits. Ladmission de réfugiés de la violence se présentant à la frontière devient une obligation de droit international et non plus un acte humanitaire ou politique discrétionnaire.

\section{Conclusion}

La proximité de crises humanitaires majeures accentuée par des interconnections croissantes et des possibilités de déplacements accrus pour les victimes de violence, l'affaiblissement des efforts des états du Nord pour assister les victimes au Sud, la fermeture des voies d'accès protégées et une asymétrie géographique croissante des droits à la protection permettent de mieux comprendre la situation des régimes d'asile actuels et la crise qui culmine en 2015-2016. Ces résultats illustrent la nécessité de prendre en compte simultanément plusieurs échelles géographiques et temporelles et de croiser les évolutions structurelles avec l'agencéité des personnes en quête de protection. Ils complètent les analyses existantes de la crise migratoire centrées sur le manque de cohérence et de solidarité des politiques européennes.

Du point de vue du droit d'asile, le contrôle à distance mené désormais par l'Europe via des accords de rétention, une surveillance accrue aux frontières et une politique générale de non-accès ${ }^{87}$, apparaît comme le revers de la médaille d'un régime d'asile tendanciellement plus ouvert à l'intérieur des territoires ${ }^{88}$. Ainsi les Etats - ou plus souvent leurs tribunaux - octroient d'une main des droits plus étendus tout en verrouillant l'accès de l'autre.

Le dénouement récent et provisoire de la crise migratoire par la fermeture de plusieurs routes d'accès à l'Europe peut être interprété à cette aune comme la victoire de la raison politique sur la raison judiciaire et comme la remise en place d'un système de mise à distance des réfugiés longtemps dominant. Laccord formel avec la Turquie de 2016 et les arrangements informels avec les factions libyennes en 2017 rappellent à cet égard l'accord passé entre l'Italie et la Libye en 2008 et la politique espagnole durant la «crise des pirogues» de $2005^{89}$.

Notre analyse s'est focalisée sur le cas européen et sur l'accroissement des demandes d'asile. Les évolutions 
structurelles que nous avons mises en évidence se manifestent cependant dans de nombreux autres contextes. Une future direction de recherche intéressante sera d'évaluer dans quelle mesure ces évolutions concernent des populations qui ne tentent pas forcément d'emprunter la voie de lasile une fois parvenue à destination (les "migrants» en général pour reprendre le terme qui s'est récemment imposé). Une autre consistera à envisager le cas de pays d'accueil non-européens tels que le Canada, les Etats-Unis, l'Afrique du sud ou l'Australie. Le Canada est à cet égard particulièrement intéressant car on y pratique de longue date des politiques alternatives et complémentaires par rapport à laccueil de demandes d’asile spontanées sur le territoire. La réinstallation ${ }^{90}$ et le sponsorship privé ${ }^{91}$ des réfugiés, peu pratiqués en Europe, sont des pistes à creuser afin de garantir une protection aux plus vulnérables sans les obliger à tenter de franchir des distances qui, si elles se réduisent, restent dramatiquement meurtrières.

\section{Notes}

$1 \mathrm{http}: / /$ ec.europa.eu/eurostat/statistics-explained/index. php/Asylum_statistics (demandes d'asile déposées pour la première fois).

2 http://cartotheque.sciences-po.fr/media/Migrants_morts_ et_disparus_en_Mediterranee_2014-2016/2268.

3 UNHCR. UNHCR Statistical Yearbook 2016. Geneva: United Nations High Commissioner for Refugees, 2017. Nous utilisons le terme "pays du Nord", au sens anglophone de Global North, pour désigner les démocraties libérales riches et "pays du Sud» au sens de Global South pour les pays moins favorisés.

4 Alexander Betts, and Paul Collier. Refuge. London: Allen Lane, 2017; Saskia Sassen. Expulsions. Paris: Gallimard NRF, 2016; H. Crawley, F. Duvell, K. Jones, S. McMahon, and N. Sigona. Destination Europe? Understanding the Dynamics and Drivers of Mediterranean Migration in 2015 (MEDMIG Final Report). University of Coventry, 2016.

5 Prem Kumar Rajaram. "Whose Migration Crisis? Editorial Introduction.» Intersections - East European Journal of Society and Politics 2, no. 4 (2016): 5-10; Heaven Crawley, and Dimitris Skleparis. «Refugees, migrants, neither, both: categorical fetishism and the politics of bounding in Europe's 'migration crisis'.» Journal of Ethnic and Migration Studies 44, no. 1 (2017): 48-64; Nicholas De Genova, ed. The Borders of Europe - Autonomy of Migration, Tactics of Bordering. Durham and London: Duke University Press, 2017; Anna Triandafyllidou. "A "Refugee Crisis" Unfolding: "Real" Events and Their Interpretation in Media and Political Debates.» Journal of Immigrant \& Refugee Studies (2017): 1-19; Nando Sigona. "The contested politics of naming in Europe's "refugee crisis".» Ethnic and Racial Studies 41, no. 3 (2018): 456-6o.
6 Kathleen Newland. «New Approaches to Refugee Crises in the 21st Century: The Role of the International Community.» In Migration Policy Institute Policy Brief 2016; Thomas Faist. "The moral polity of forced migration.» Ethnic and Racial Studies 41, no. 3 (2018): 412-23; Wolfgang Streeck. «Exploding Europe: Germany, the Refugees and the British Vote to Leave.» SPERI Paper - University of Sheffield, no. 31 (2016); Leo Lucassen. "Peeling an onion: the "refugee crisis" from a historical perspective.» Ethnic and Racial Studies 41, no. 3 (2017): 383-410.

7 Nicolas Jacob-Rousseau. "Géohistoire/géo-histoire: quelles méthodes pour quel récit?». Géocarrefour 84, no. 4 (2009): 211-16.

8 "(...) narratives about how things got 'from here to there' including the multiple contingencies and reversals encountered in the process. At this level of analysis, it is possible to delineate, at least partially, the structural constraints and other obstacles affecting a specific individual or collective pursuit." Alejandro Portes. "The hidden abode: Sociology as analysis of the unexpected». American Sociological Review 65, no. 2 (2000): 1-18.

9 Bundy identifie quatre "crises de l'asile» historiques en Europe: la révolution russe et la 1e guerre mondiale, la 2e guerre mondiale et ses suites, les années 9o (ex-Yougoslavie, etc.) \& la période actuelle dès 2011. Colin Bundy. «Migrants, réfugiés, histoire et précèdents.» Revue des migrations forcées no. 1 (2016): 5-6.

10 Un certain nombre de demandes enregistrées en 2016 ont été déposées en 2015, année qui marque l’apogée des arrivées. Cf. EAso. Annual Report on the Situation of Asylum in the European Union 2016. Cyprus: European Asylum Support Office, 2017.

11 Klaus J. Bade. L'Europe en mouvement - La migration de la fin du xvirIe siècle à nos jours. Paris: Seuil, 2002.

12 Benedicte Michalon et Mihaela Nedelcu. «Histoire, constantes et transformations récentes des dynamiques migratoires en Roumanie.» Revue d'Etudes Comparatives EstOuest 41, no. 4 (2010): 5-28.

13 Le HCR estime que la moitié des demandes d'asile de 1992 émanaient de Roumains et de Bulgares ayant peu de motifs d'asile au sens de la Convention de 1951. Cf. UNHCR. «Background Paper on Refugees and Asylum Seekers from Romania.» Geneva: Un High Commissioner for Refugees, 1994; HCR. Les réfugiés dans le monde 2000 - Cinquante ans d'action humanitaire. Paris: Autrement - Haut Commissariat des Nations Unies pour les réfugiés, 2000.

14 Gil Loescher. The UNHCR and world politics - A perilous path. Oxford: Oxford University Press, 2001.

15 Bade, L'Europe en mouvement - La migration de la fin $d u$ XVIIIe siècle à nos jours.

$16 \mathrm{HCR}$, Les réfugiés dans le monde 2000 - Cinquante ans d'action humanitaire.

17 Bade, L'Europe en mouvement - La migration de la fin du xvirle siècle à nos jours. 
$18 \mathrm{HCR}$, Les réfugiés dans le monde 2000 - Cinquante ans d'action humanitaire.

19 François Héran. Le temps des immigrés. Paris: Seuil, 2007.

20 Bruno Marnot. Les migrations internationales en Europe et aux Etats-Unis des années 1840 à 1940. Neuchâtel: Alphil, 2006.

21 Crawley and Skleparis, «Refugees, migrants, neither, both: categorical fetishism and the politics of bounding in Europe's 'migration crisis'."

22 Timothy J Hatton, and Joe Moloney. «Applications for Asylum in the Developed World: Modelling Asylum Claims by Origin and Destination.» ANU Working Papers in Economics and Econometrics, no. 625 (2015): 1-27.

23 Gérard-François Dumont. "L'Ue face à l'immigration.» Bulletin de liaison de la Société de géographie, no. 29 (2016): 55-62.

24 Hélène Thiollet. «Wad Sharifey, Kishm el-Girbâ, Asotriba... Métamorphoses d'un réseau régional de douze camps de réfugiés érythréens dans l'est du Soudan (1962-2013).» In Un monde de camps, edited by Michel Agier, 203-17. Paris: La Découverte, 2014.

25 Richard Black, and Vaughan Robinson. Geography and refugees. London: Belhaven Press, 1993.

26 Will H. Moore, and Stephen M. Shellman. «Whither Will They Go? A Global Study of Refugees' Destinations, 19651995.»International Studies Quarterly 51, no. 4 (2007): 811-34.

27 Christian Grataloup. Introduction à la géohistoire. Paris: Armand Colin, 2015.

28 La Convention stipule dans son article 1 (al. 2) que seuls les événements survenus avant 1951 en Europe peuvent être pris en considération pour la reconnaissance de l'asile à une personne craignant «avec raison dêtre persécutée du fait de sa race, de sa religion, de sa nationalité, de son appartenance à un certain groupe social ou de ses opinions politiques». Le protocole de 1967 élargi la protection aux événements survenus après 1951 et au dehors de l'Europe. http://www.unhcr.org/fr/about-us/backgroun$\mathrm{d} / 4 \mathrm{~b} 14 \mathrm{f} 4 \mathrm{a} 62 /$ convention-protocole-relatifs-statut-refugies. html.

29 Loescher, The UNHCR and world politics - A perilous path.

30 John Hope Simpson. "The Refugee Problem.» International Affairs 17, no. 5 (1938): 607-28.

$31 \mathrm{http}: / /$ news.psu.edu/story/350156/2015/03/26/research/istresearchers-explore-technology-use-syrian-refugee-camp.

$32 \mathrm{http}: / /$ data.worldbank.org/indicator/IT.CEL.SETS.P2.

33 Marie McAuliffe. "The appification of migration.» Asia \& the Pacific Policy Society - Policy Forum, no. 20 (2016); Judith Zijlstra, and Ilse Van Liempt. «Smart(phone) travelling: understanding the use and impact of mobile technology on irregular migration journeys.» International Journal of Migration and Border Studies 3, no. 2-3 (2017): 174-91.

34 Virginia Signorini. "Le téléphone satellite au secours des réfugiés." Revue des migrations forcées, no. 38 (2011): 24; Rianne Dekker, and Godfried Engbersen. «How social media transform migrant networks and facilitate migration.» Global Networks: A Journal of Transnational Affairs 14, no. 4 (2014): 401-18.

$35 \mathrm{https}: / /$ play.google.com/store/apps/details ?id=com. dvuckovic.asylumseeker.

$36 \mathrm{https://play.google.com/store/apps/details?id=com.mem-}$ orado.welcomeGuide, https://arriving-in-berlin.de/.

37 https:/www.newsdeeply.com/refugees/community/2017/ 02/20/a-translator-in-your-pocket-app-aims-to-bridgerefugees-language-gap.

38 Kochhar Ranesh. A Global Middle Class Is More Promise than Reality. Pew Research Report 2015. Pour des données complémentaires, cf. https://ourworldindata.org/.

39 Arjun Appadurai. "The Capacity to Aspire: Culture and the Terms of Recognition.» In Culture and Public Action, edited by V. Rao and M. Walton, 59-84. Palo Alto: Stanford University Press, 2004.

40 Pinar Yazgan, Deniz Eroglu Utku, and Ibrahim Sirkeci. «Syrian Crisis and Migration.» Migration Letters 12, no. 3 (2015) : 181-92.

41 Catherine Wihtol de Wenden. Le droit démigrer. Paris: CNRS / Débats, 2013.

42 https://www.sem.admin.ch/sem/fr/home/asyl/eritrea/faq. html.

43 Durant la guerre froide, les Etats occidentaux sont opposés aux rapatriements. Le départ est vu comme la preuve de léchec des états communistes. Cette période est désignée dans la littérature comme celle de l'«exilic bias», cf. Matthew E. Price. Rethinking Asylum. Cambridge: Cambridge University Press, 2009. ou de l'« exilic approach », cf. Alexander Aleinikoff. «State-Centered Refugee Law: From Resettlement to Containment.» Mich. J. Int'l L. 14, no. 1 (1992): 120-38.

44 Price, Rethinking Asylum.

45 Rachel Landry. "The "humanitarian smuggling» of refugees: criminal offence or moral obligation.» Refugee Studies Center working paper, no. 119 (2016).

46 Luc Legoux. "La réorganisation mondiale de l'asile.» In Lasile au sud, edited by Luc Cambrézy, Smaïn Laacher, Véronique Lassailly-Jacob and Luc Legoux. Paris: La Dispute, 2008.

47 Gil Loescher. «UNHCR’s Origins and Early History: Agency, Influence and Power in Global Refugee Policy.» Refuge 33, no. 1 (2017): 77-86.

48 Jeff Crisp. «A new asylum paradigm: globalization, migration and the uncertain future of the international refugee regime.» New Issues in Refugee Research - UNHCR, no. 100 (2003).

49 B. S. Chimni. "Aid, Relief, and Containment: The First Asylum Country and Beyond.» International Migration 40, no. 5 (2002): 75-94; Andrew Shacknove. «From Asylum to Containment.» International Journal of Refugee Law 5, no. 4 (1993): 516-33; Aleinikoff, «State-Centered Refugee Law: From Resettlement to Containment.» 
50 B.S. Chimni. "The Geopolitics of Refugee Studies: A View from the South. " Journal of Refugee Studies 11, no. 4 (1998): 350-74.

51 Pour les Syriens et malgré les arrivées en Europe de 2015/6, le ratio reste de 5 réfugiés dans les pays limitrophes contre 1 en Europe. De même, l'Ethiopie abrite 734 ooo réfugiés du Sud-Soudan, de Somalie et d'Erythrée. Source: http :// www.unhcr.org/56b3251917.html.

52 Crisp, "A new asylum paradigm: globalization, migration and the uncertain future of the international refugee regime."

53 Guy S. Goodwin-Gill. «The Mediterranean Papers: Athens, Naples, and Istanbul. » International Journal of Refugee Law 28, no. 2 (2016): 276-309.

54 Crisp, "A new asylum paradigm: globalization, migration and the uncertain future of the international refugee regime.»; Gil Loescher, Alexander Betts, and James Milner. The United Nations High Commissioner for Refugees (UNHCR): the politics and practice of refugee protection into the twenty-first century. London: Routledge, 2008.

55 http://www.refugeesinternational.org/reports/2015/10/4/adaily-struggle-to-survive-syrian-refugees-in-lebanon.

56 http://www.unhcr.org/fr/558adcc38.html.

57 Catherine-Lune Grayson et François Audet. «Les hauts et les bas du financement canadien au HCR: quelle aide et pour quels réfugiés?». Refuge 33, no. 1 (2017) : 62-76.

58 W. Courtland Robinson. "The Comprehensive Plan of Action for Indochinese Refugees, 1989-1997: Sharing the Burden and Passing the Buck. » Journal of Refugee Studies 17 , no. 3 (2004): 319-33.

59 Crisp, «A new asylum paradigm: globalization, migration and the uncertain future of the international refugee regime.»

60 Matthew J. Gibney. «The state of asylum: democratization, judicialization and evolution of refugee policy in Europe.» Ibid., no. 50 (2001).

61 Loescher, The UNHCR and world politics - A perilous path. La mise en place de "safe zones» au Sri-Lanka en 2009 s'avéra elle aussi problématique. Cette voie reste cependant évoquée (Syrie, Myanmar) et pourrait réapparaître avec le rétablissement de la rétention.

62 Ce statut n'a cependant jamais véritablement été ancré dans le droit international, cf. R. Värk. «Diplomatic Asylum: Theory, Practice and the Case of Julian Assange. " Proceedings of the Estonian Academy of Security Sciences, no. 11 (2012) : 240-57.

63 Robinson, "The Comprehensive Plan of Action for Indochinese Refugees, 1989-1997: Sharing the Burden and Passing the Buck. »

64 Loescher, The UNHCR and world politics - A perilous path.

65 Susan Fratzke, and Brian Salant. Tracing the Channels Refugees Use to Seek Protection in Europe. Brussels: Migration Policy Institute Europe Policy Brief 2017. Pour les chiffres des réinstallations, cf. http://www.unhcr.org/resettlement. html.
66 G. Noll, J. Fagerlund, and F. Liebaut. Study on the Feasibility of Processing Asylum Claims Outside the EU against the Background of the Common European Asylum System and the Goal of a Common Asylum Procedure. Luxemburg: European Commission, 2002. Dans de nombreux pays l'asile diplomatique était restreint aux réfugiés politiques au sens étroit. Cependant, dans le cas de la Suisse p.ex., il a concerné un éventail plus large et des effectifs considérables de personnes. Cf. Aline Catzeflis. La suppression des demandes d'asile en ambassades suisses. Neuchâtel: Université de Neuchâtel (Mémoire de Master), 2016.

67 La Suisse a été la dernière à remplacer la demande d'asile en ambassade par une procédure beaucoup plus restrictive de visa humanitaire en 2014.

68 Gibney, «The state of asylum: democratization, judicialization and evolution of refugee policy in Europe.»

69 Eiko Thielemann, and Mogens Hobolth. "Trading numbers vs. rights? Accounting for liberal and restrictive dynamics in the evolution of asylum and refugee policies. " Journal of Ethnic and Migration Studies 42, no. 4 (2016): 643-64. La tendance schizophrénique des politiques d'asile à se durcir d'un côté tout en étant plus accommodantes de l'autre est confirmée par des indices de restrictivité agrégés remarquablement stables dans la plupart des pays d'accueil, cf. Samuel D. Schmid, and Marc Helbling. "Validating the Immigration Policies in Comparison (IMPIC) Dataset.» WZB Berlin Social Science Center Discussion Paper, no. SP $\mathrm{VI} / 2 \mathrm{O} 2$ (2016).

70 Claudena M. Skran. «The International Refugee Regime: The Historical and Contemporary Context of International Responses to Asylum Problems. » In Refugees and the Asylum Dilemma in the West, edited by Gil Loescher, 8-35: Pennsylvania State University, 1992.

71 Matthew J. Gibney. "Asylum Principled Hypocrisy.» In Migration: A COMPAS Anthology (on line), edited by B. Anderson and M. Keith. Oxford: COMPAs, 2015.

72 UnHCR. Guidelines on International Protection 12. Geneva: United Nations High Commissioner for Refugees, 2016.

73 Loescher, The UNHCR and world politics - A perilous path.

$74 \mathrm{HCR}$, Les réfugiés dans le monde 2000 - Cinquante ans d'action humanitaire; Arthur C. Helton. The price of indifference. Refugees and Humanitarian Action in the New Century. Oxford: Oxford University Press, 2002.

75 HCR, Les réfugiés dans le monde 2000 - Cinquante ans d'action humanitaire.

76 EAso, Annual Report on the Situation of Asylum in the European Union 2016.

77 Gibney, «The state of asylum: democratization, judicialization and evolution of refugee policy in Europe.»

78 Etablir si les questions liées à l'asile se dépolitisent est délicat. A l'échelle internationale, on observe une dépolitisation (l'asile n'est plus un enjeu diplomatique (high-politics)), de même quà l'échelle individuelle (élargissement des motifs). Mais simultanément, une politisation se manifeste car 
l'asile devient une question débattue en politique intérieure («low-politics»).

79 Price, Rethinking Asylum.

$80 \mathrm{HCR}$, Les réfugiés dans le monde 2000 - Cinquante ans d'action humanitaire.

81 Alexander Betts. «Survival Migration: A New Protection Framework.» Global Governance 16, no. 3 (2010) : 361-82.

82 Goepfert Salome. «Schutz für Flüchtlinge bewaffneter Konflikte.» Jusletter, no. 18 (2016) : 2-21.

83 Chimni, "The Geopolitics of Refugee Studies: A View from the South»; Elspeth Guild, and Didier Bigo. «Le visa Schengen: expression d'une stratégie de "police» à distance.» Cultures et Conflits 1, no. 49 (2003): 22-37.

84 Price, Rethinking Asylum.

85 https://curia.europa.eu/jcms/upload/docs/application/ pdf/2017-03/cp170024en.pdf

86 Salome, «Schutz für Flüchtlinge bewaffneter Konflikte.»

87 Guild and Bigo, «Le visa Schengen: expression d'une stratégie de "police» à distance»; Thielemann and Hobolth, «Trading numbers vs. rights? Accounting for liberal and restrictive dynamics in the evolution of asylum and refugee policies. »

88 Matthew J. Gibney, and Randall Hansen. «Asylum Policy in the West: Past Trends, Future Possibilities.» United Nations University - WIDER - Discussion Paper, no. 68 (2003).

89 Luna Vives. «Unwanted sea migrants across the eu border: The Canary Islands.» Political Geography 61, no. Supplement C (2017): 181-92.

90 Michael Casasola. "The Indochinese Refugee Movement and the Subsequent Evolution of UNHCR and Canadian Resettlement Selection Policies and Practices.» Refuge 32, no. 2 (2016): 41-53.

91 Michael J. Molloy, and James C. Simeon. «The Indochinese Refugee Movement and the Launch of Canada's Private Sponsorship Program.» Refuge 32, no. 2 (2016) : 3-8.

Étienne Piguet est chercheur à l'Institut de Géographie Université de Neuchâtel (Suisse). L'auteur peut être contacté à Etienne .piguet@unine.ch. 